Article

\title{
Strongly Convex Functions of Higher Order Involving Bifunction
}

\author{
Bandar B. Mohsen ${ }^{1}$, Muhammad Aslam Noor ${ }^{2}{ }^{\mathbb{D}}$, Khalida Inayat Noor $^{2}{ }^{(1)}$ and \\ Mihai Postolache $3,4, *$ (D) \\ 1 Department of Mathematics, College of Science, King Saud University, Riyadh 11451, Saudi Arabia; \\ balmohsen@ksu.edu.sa \\ 2 Department of Mathematics, COMSATS University Islamabad, Islamabad 45550, Pakistan; \\ noormaslam@gmail.com (M.A.N.); khalidan@gmail.com (K.I.N.) \\ 3 Center for General Education, China Medical University, Taichung 40402, Taiwan \\ 4 Department of Mathematics and Informatics, University Politehnica of Bucharest, 060042 Bucharest, Romania \\ * Correspondence: mihai@mathem.pub.ro
}

Received: 28 September 2019; Accepted: 26 October 2019; Published: 1 November 2019

\begin{abstract}
Some new concepts of the higher order strongly convex functions involving an arbitrary bifuction are considered in this paper. Some properties of the higher order strongly convex functions are investigated under suitable conditions. Some important special cases are discussed. The parallelogram laws for Banach spaces are obtained as applications of higher order strongly affine convex functions as novel applications. Results obtained in this paper can be viewed as refinement and improvement of previously known results.
\end{abstract}

Keywords: convex function; monotone operator; strongly convex function

MSC: 26D25; 49J40; 90C33

\section{Introduction}

Lin and Fukushima [1] introduced the concept of higher order strongly convex functions and used it in the study of mathematical program with equilibrium constraints. Mishra and Sharma [2] derived the Hermite-Hadamard type inequalities for higher order strongly convex functions.

To be more precise, a function $F$ on the convex and closed set $K$ is said to be a higher order strongly convex, if there exists a constant $\mu \geq 0$. such that

$$
F((1-t) u+t v) \leq(1-t) F(u)+t F(v)-\mu \varphi(t)\|v-u\|^{p}
$$

for all $u, v \in K, t \in[0,1], p \geq 1$, where

$$
\varphi(t)=t(1-t) .
$$

If $p=2$, then higher order strongly convex functions become strongly convex functions with the same $\varphi(t)$ as defined in (1). That is,

$$
F((1-t) u+t v) \leq(1-t) F(u)+t F(v)-\mu t(1-t)\|v-u\|^{2}, \forall u, v \in K, t \in[0,1] .
$$


We would like to point out that the strongly convex functions were introduced and studied by Polyak [3], which play an important part in optimization theory and related areas. For example, Karmardian [4] used the strongly convex functions to discuss the unique existence of a solution of the nonlinear complementarity problems. Strongly convex functions also played an important role in the convergence analysis of the iterative methods for solving variational inequalities and equilibrium problems, see $\mathrm{Zu}$ and Marcotte [5]. Nikodem and Pales [6] investigated the characterization of the inner product spaces using the strongly convex functions, which can be viewed as a novel and innovative application. Qu and Li [7] investigated the exponentially stability of primal-dual gradient dynamics using the concept of strongly convex functions. Awan et al. [8] have derived Hermite-Hadamard type inequalities for various classes of strongly convex functions, which provide upper and lower estimates for the integrand. For more applications and properties of the strongly convex functions, see References [1-18] and the references therein.

We have noticed that the function $\varphi(\cdot)$ in (1) is not correct and must be modified. Characterizations of the higher order strongly convex functions discussed in Lin and Fukushima [1] are not correct. These facts and observations inspired us to consider higher order strongly convex functions involving an arbitrary bifuction $\xi(\cdot, \cdot)$. New concepts of monotonicity are introduced. New results under some mild conditions have been obtained. Our results represent the refinement and improvement of the results of Lin and Fukushima [1]. Higher order strongly convex functions can be used to characterize the uniformly reflex Banach spaces. We have also deduced the weakly parallelogram laws for the $L^{p}$-spaces, which have been discussed in References [19-23] from the concept of higher order strongly affine convex functions. This fact can be viewed as an elegant and interesting application of the higher order strongly convex functions. As special cases, one can obtain various new and refined versions of known results. It is expected that the ideas and techniques of this paper may stimulate further research in this field.

\section{Formulations and Basic Facts}

Let $K$ be a nonempty set in a real Hilbert space $H$. The inner product and norm are denoted by by $\langle\cdot, \cdot\rangle$ and $\|\cdot\|$, respectively.

Definition 1 ([1,7]). A set $K$ in $H$ is said to be a convex set if

$$
u+t(v-u) \in K, \quad \forall u, v \in K, t \in[0,1] .
$$

We now introduce some new classes of higher order strongly convex functions and higher order strongly affine convex functions with respect to the bifunction $\xi(\cdot, \cdot)$.

Definition 2. A function $F$ on the convex set $K$ is said to be higher order strongly convex with respect to the bifunction $\xi(\cdot, \cdot)$ if there exists a constant $\mu>0$, such that

$$
F(u+t(v-u)) \leq(1-t) F(u)+t F(v)-\mu\left\{t^{p}(1-t)+t(1-t)^{p}\right\}\|\xi(v, u)\|^{p}, p>0 .
$$

for all $u, v \in K, t \in[0,1]$.

A function $F$ is said to higher order strongly concave with respect to bifunction $\xi(\cdot, \cdot)$, if and only if, $-F$ is higher order strongly convex $\xi(\cdot, \cdot)$.

If $t=\frac{1}{2}$, then

$$
F\left(\frac{u+v}{2}\right) \leq \frac{F(u)+F(v)}{2}-\mu \frac{1}{2^{p}}\|\xi(v, u)\|^{p}, P>0, \quad \forall u, v \in K
$$


The function $F$ is said to be a higher order strongly $J$-convex function.

We now discuss some special cases.

(I). If $p=2$, then the higher order strongly convex function becomes strongly convex functions, that is,

$$
F(u+t(v-u)) \leq(1-t) F(u)+t F(v)-\mu t(1-t)\|\xi(v, u)\|^{2}, \quad \forall u, v \in K, t \in[0,1] .
$$

For the properties of the strongly convex functions in variational inequalities and equilibrium problems, see Noor [2,6] and Oettli et al. [17].

(II). If $\xi(v, u)=v-u$, then Definition 2 reduces to

Definition 3. A function $F$ fn the convex set $K$ is said to be higher order strongly convex if there exists a constant $\mu>0$, such that

$$
F(u+t(v-u)) \leq(1-t) F(u)+t F(v)-\mu\left\{t^{p}(1-t)+t(1-t)^{p}\right\}\|v-u\|^{p}, p>0,
$$

for all $u, v \in K, t \in[0,1]$.

This notion was introduced and studied by Noor and Noor [16].

(III). If $\xi(v, u)=h(v-u)$, then Definition 2 reduces to

Definition 4. A function $F$ on the convex set $K$ is said to be higher order strongly convex with respect to an arbitrary function $h(\cdot)$, if there exists a constant $\mu>0$ such that

$$
F(u+t(v-u)) \leq(1-t) F(u)+t F(v)-\mu\left\{t^{p}(1-t)+t(1-t)^{p}\right\}\|h(v-u)\|^{p}, p>0,
$$

for all $u, v \in K, t \in[0,1]$.

The above notion appears to be new one. For $p=2$, we have the strongly convex functions, introduced and studied by Ademak [9].

For appropriate and suitable choice of the bifunction $\xi(\cdot, \cdot)$ and $p$ one can obtain various new and known classes of strongly convex functions. This shows that the higher order strongly convex functions involving the bifunction $\xi(\cdot, \cdot)$ is quite a general and unifying one. One can explore the applications of the higher order strongly convex functions, this is another direction for further research.

Definition 5. A function $F$ on the convex set $K$ is said to be a higher order strongly affine convex with respect to the bifunction $\xi(\cdot, \cdot)$ if there exists a constant $\mu>0$ such that

$$
F(u+t(v-u))=(1-t) F(u)+t F(v)-\mu\left\{t^{p}(1-t)+t(1-t)^{p}\right\}\|\xi(v, u)\|^{p}, p>0,
$$

for all $u, v \in K, t \in[0,1]$.

Note that, if a function is both higher order strongly convex and higher order strongly concave, then it is a higher order strongly affine convex function.

A function $F$ is called a higher order strongly quadratic equation with respect to the bifunction $\xi(\cdot, \cdot)$ if there exists a constant $\mu>0$ such that

$$
F\left(\frac{u+v}{2}\right)=\frac{F(u)+F(v)}{2}-\mu \frac{1}{2^{p}}\|\xi(v, u)\|^{p}, p>0,
$$


for all $u, v \in K, t \in[0,1]$. This function $F$ is also called a higher order strongly affine $J$-convex function.

Definition 6. A function $F$ on the convex set $K$ is said to be higher order strongly quasi convex, if there exists a constant $\mu>0$ such that

$$
F\left(u+t(v-u) \leq \max \{F(u), F(v)\}-\mu\left\{t^{p}(1-t)+t(1-t)^{p}\right\}\|\xi(v, u)\|^{p}, p>0,\right.
$$

for all $u, v \in K, t \in[0,1]$.

Definition 7. A function $F$ on the convex set $K$ is said to be higher order strongly log-convex, if there exists a constant $\mu>0$ such that

$$
F(u+t(v-u)) \leq(F(u))^{1-t}(F(v))^{t}-\mu\left\{t^{p}(1-t)+t(1-t)^{p}\right\}\|\xi(v, u)\|^{p}, p>0,
$$

for all $u, v \in K, t \in[0,1]$. where $F(\cdot)>0$.

From the above definitions, we have

$$
\begin{aligned}
F(u+t(v-u)) & \leq(F(u))^{1-t}(F(v))^{t}-\mu\left\{t^{p}(1-t)+t(1-t)^{p}\right\}\|\xi(v, u)\|^{p} \\
& \leq(1-t) F(u)+t F(v)-\mu\left\{t^{p}(1-t)+t(1-t)^{p}\right\}\|\xi(v, u)\|^{p} \\
& \leq \max \{F(u), F(v)\}-\mu\left\{t^{p}(1-t)+t(1-t)^{p}\right\}\|\xi(v, u)\|^{p}
\end{aligned}
$$

This shows that every higher order strongly log-convex function is a higher order strongly convex function and every higher order strongly convex function is a higher order quasi-convex function. However, the converse is not true.

Definition 8. An operator $T: K \rightarrow H$ is said to be:

1. higher order strongly monotone, if and only if there exists a constant $\alpha>0$ such that

$$
\langle T u-T v, u-v\rangle \geq \alpha\|\xi(v, u)\|^{p}, p>0, \quad \forall u, v \in K .
$$

2. higher order strongly pseudomonotone if and only if there exists a constant $v>0$ such that

$$
\langle T u, v-u\rangle+v\|\xi(v, u)\|^{p}, P>0, \geq 0 \Rightarrow\langle T v, v-u\rangle \geq 0, \quad \forall u, v \in K .
$$

3. higher order strongly relaxed pseudomonotone if and only if there exists a constant $\mu>0$ such that

$$
\langle T u, v-u\rangle \geq 0 \quad \Rightarrow \quad-\langle T v, u-v\rangle+\mu\|\xi(v, u)\|^{p}, \geq 0, \quad \forall u, v \in K, p>0 .
$$

Definition 9. A differentiable function $F$ on the convex set $K$ is said to be higher order strongly pseudo convex function if and only if there exists a constant $\mu>0$ such that

$$
\left\langle F^{\prime}(u), v-u\right\rangle+\mu\|\xi(v, u)\|^{p} \geq 0 \Rightarrow F(v) \geq F(u), \quad \forall u, v \in K .
$$

We also need the following assumptions regarding the bifunction $\xi(\cdot, \cdot)$.

Condition $\mathbf{N}$. The bifunction $\xi(\cdot, \cdot)$ is required to satisfy this assumption:

$$
\xi\left(u+t_{1}(v-u), u+t_{2}(v-u)\right)=\left(t_{1}-t_{2}\right) \xi(v, u), \quad \forall u, v \in K .
$$


If $t_{1}=0$, then the Definition 2 becomes

$$
\xi\left(u, u+t_{2}(v-u)\right)=-t_{2} \xi(v, u) . \quad \forall u, v \in K .
$$

and if $t_{1}=1$, then the Definition 2 become

$$
\xi\left(v, u+t_{2}(v-u)\right)=\left(1-t_{2}\right) \xi(v, u), \quad \forall u, v \in K .
$$

\section{Main Results}

In this section, we consider some basic properties of higher order strongly convex functions.

Theorem 1. Let $F$ be a differentiable function on the convex set $K$ and condition $N$ hold. Then the function $F$ is higher order strongly convex function, if and only if,

$$
F(v)-F(u) \geq\left\langle F^{\prime}(u), v-u\right\rangle+\mu\|\xi(v, u)\|^{p}, \quad \forall v, u \in K .
$$

Proof. Let $F$ be a higher order strongly convex function on the convex set $K$. Then

$$
F(u+t(v-u)) \leq(1-t) F(u)+t F(v)-\mu\left\{t^{p}(1-t)+t(1-t)^{p}\right\}\|\xi(v, u)\|^{p}, \quad \forall u, v \in K,
$$

which can be written as

$$
F(v)-F(u) \geq \frac{F(u+t(v-u)-F(u)}{t}+\left\{t^{p-1}(1-t)+(1-t)^{p}\right\}\|\xi(v, u)\|^{p} .
$$

Taking the limit in the above inequality as $t \rightarrow 0$, we have

$$
F(v)-F(u) \geq\left\langle F^{\prime}(u), v-u\right\rangle+\mu\|\xi(v, u)\|^{p}, \quad \forall u, v \in K .
$$

which is (2), the required result.

Conversely, let (2) hold. Then, for all $u, v \in K, t \in[0,1], v_{t}=u+t(v-u) \in K$, and using Condition $\mathrm{N}$, we have

$$
\begin{aligned}
F(v)-F\left(v_{t}\right) & \geq\left\langle F^{\prime}\left(v_{t}\right), v-v_{t}\right\rangle+\mu\left\|\xi\left(v, v_{t}\right)\right\|^{p} \\
& =(1-t)\left\langle F^{\prime}\left(v_{t}\right), v-u\right\rangle+\mu(1-t)^{p}\|\xi(v, u)\|^{p}, \quad \forall u, v \in K
\end{aligned}
$$

In a similar way, we obtain

$$
\begin{aligned}
F(u)-F\left(v_{t}\right) & \geq\left\langle F^{\prime}\left(v_{t}\right), u-v_{t}\right\rangle+\mu\left\|\xi\left(u, v_{t}\right)\right\|^{p} \\
& =-t\left\langle F^{\prime}\left(v_{t}\right), v-u\right\rangle+\mu t^{p}\|\xi(v, u)\|^{p} .
\end{aligned}
$$

Multiplying (3) by $t$ and (4) by $(1-t)$ and adding the resultant, we have

$$
F(u+t \eta(v, u)) \leq(1-t) F(u)+t F(v)-\mu\left\{t^{p}(1-t)+t(1-t)^{p}\right\}\|\xi(v, u)\|^{p}, \quad \forall u, v \in K,
$$

showing that $F$ is a higher order strongly convex function.

Theorem 2. Let $F$ be a differentiable higher order strongly convex function on the convex set $K$. Then

$$
\left\langle F^{\prime}(u)-F^{\prime}(v), u-v\right\rangle \geq \mu\left\{\|\xi(v, u)\|^{p}+\|\xi(u, v)\|^{p}\right\}, \quad \forall u, v \in K .
$$


Proof. Let $F$ be a higher order strongly convex function on the convex set $K$. Then, from Theorem 1 . we have

$$
F(v)-F(u) \geq\left\langle F^{\prime}(u), v-u\right\rangle+\mu\|\xi(v, u)\|^{p}, \quad \forall u, v \in K .
$$

Changing the role of $u$ and $v$ in (5), we have

$$
F(u)-F(v) \geq\left\langle F^{\prime}(v), u-v\right\rangle+\mu\|\xi(v, u)\|^{p}, \quad \forall u, v \in K .
$$

Adding (5) and (6), we have

$$
\left\langle F^{\prime}(u)-F^{\prime}(v), u-v\right\rangle \geq \mu\left\{\|\xi(v, u)\|^{p}+\|\xi(u, v)\|^{p}\right\}, \quad \forall u, v \in K .
$$

which shows that $F^{\prime}($.$) is a higher order strongly monotone operator.$

We remark that the converse of Theorem 2 is not true. In this direction, we have the following result.

Theorem 3. If the differential operator $F^{\prime}(\cdot)$ of a differentiable higher order strongly convex function $F$ is a higher order strongly monotone operator and Condition $N$ holds, then

$$
F(v)-F(u) \geq\left\langle F^{\prime}(u), v-u\right\rangle+2 \mu \frac{1}{p}\|\xi(v, u)\|^{p}, \quad \forall u, v \in K .
$$

Proof. Let $F^{\prime}$ be a higher order strongly monotone operator. Then, from (7), we have

$$
\left.\left\langle F^{\prime}(v), u-v\right\rangle \geq\left\langle F^{\prime}(u), u-v\right)\right\rangle+\mu\left\{\|\xi(v, u)\|^{p}+\|\xi(u, v)\|^{p}\right\}, \quad \forall u, v \in K .
$$

Since $K$ is an convex set, for all $u, v \in K, t \in[0,1], v_{t}=u+t(v-u) \in K$. Taking $v=v_{t}$ in ((9) and using Condition $\mathrm{N}$, we have

$$
\begin{aligned}
\left\langle F^{\prime}\left(v_{t}\right), u-v_{t}\right\rangle & \leq\left\langle F^{\prime}(u), u-v_{t}\right\rangle-2 \mu\left\{\left\|\xi\left(v_{t}, u\right)\right\|^{p}+\left\|\xi\left(u, v_{t}\right)\right\|^{p}\right\} \\
& =-t\left\langle F^{\prime}(u), v-u\right\rangle-2 \mu t^{p}\|\xi(v \cdot u)\|^{p}
\end{aligned}
$$

which implies that

$$
\left\langle F^{\prime}\left(v_{t}\right), v-u\right\rangle \geq\left\langle F^{\prime}(u), v-u\right\rangle+2 \mu t^{p-1}\|\xi(v, u)\|^{p}
$$

Consider the auxiliary function $\zeta(t)=F(u+t(v-u)$, for all $u, v \in K$, from which we get $\zeta(1)=F(v)$ and $\zeta(0)=F(u)$. Then, from $(10)$, we have

$$
\zeta^{\prime}(t)=\left\langle F^{\prime}\left(v_{t}\right), v-u\right\rangle \geq\left\langle F^{\prime}(u), v-u\right\rangle+2 \mu t^{p-1}\|\xi(v, u)\|^{p} .
$$

Integrating (11) between 0 and 1, we have

$$
\begin{aligned}
\zeta(1)-\zeta(0)= & \int_{0}^{1} \zeta^{\prime}(t) d t \\
& \geq\left\langle F^{\prime}(u), v-u\right\rangle+2 \mu \frac{1}{p}\|\xi(v, u)\|^{p} .
\end{aligned}
$$


Thus it follows that

$$
F(v)-F(u) \geq\left\langle F^{\prime}(u), v-u\right\rangle+2 \mu \frac{1}{p}\|\xi(v, u)\|^{p}, \forall u, v \in K,
$$

which is the required (8).

We note that if $p=2$ then Theorem 3 can be viewed as the converse of Theorem 2 .

We now give a necessary condition for a higher order strongly pseudo-convex function.

Theorem 4. Let $F^{\prime}(\cdot)$ be a higher order strongly relaxed pseudomonotone operator and condition $N$ holds. Then $F$ is a higher order strongly pseudo-connvex function.

Proof. Let $F^{\prime}(\cdot)$ be a higher order strongly relaxed pseudomonotone operator. Then, $\forall u, v \in K$,

$$
\left\langle F^{\prime}(u), v-u\right\rangle \geq 0 .
$$

implies that

$$
\left\langle F^{\prime}(v), v-u\right\rangle \geq \mu\|\xi(v \cdot u)\|^{p}, \quad \forall u, v \in K
$$

Since $K$ is an convex set, $\forall u, v \in K, \quad t \in[0,1], v_{t}=u+t(v-u) \in K$.

Taking $v=v_{t}$ in (12) and using Condition $\mathrm{N}$, we have

$$
\left\langle F^{\prime}\left(v_{t}\right), v-u\right\rangle \geq \mu t^{p-1}\|\xi(v \cdot u)\|^{p} .
$$

Consider the auxiliary function

$$
\zeta(t)=F(u+t(v-u))=F\left(v_{t}\right), \quad \forall u, v \in K, t \in[0,1],
$$

which is differentiable, since $F$ is differentiable function. Then, using (13), we have

$$
\left.\zeta^{\prime}(t)=\left\langle F^{\prime}\left(v_{t}\right), v-u\right)\right\rangle \geq \mu t^{p-1}\|\xi(v, u)\|^{p} .
$$

Integrating the above relation between 0 to 1 , we obtain

$$
\zeta(1)-\zeta(0)=\int_{0}^{1} \zeta^{\prime}(t) d t \geq \frac{\mu}{p}\|\xi(v, u)\|^{p}
$$

that is,

$$
\left.F(v)-F(u) \geq \frac{\mu}{p}\|\xi(v, u)\|^{p}\right), \forall u, v \in K,
$$

showing that $F$ is a higher order strongly pseudo-convex function.

Definition 10. A function $F$ is said to be sharply higher order strongly pseudo convex, if there exists a constant $\mu>0$ such that

$$
\left\langle F^{\prime}(u), v-u\right\rangle \geq 0 \quad \Rightarrow \quad F(v) \geq F(v+t(u-v))+\mu\left\{t^{p}(1-t)+t(1-t)^{p}\right\}\|\xi(v, u)\|^{p}, p>0,
$$

for all $u, v \in K, t \in[0,1]$. 
Theorem 5. Let $F$ be a sharply higher order strongly pseudo convex function on $K$ with a constant $\mu>0$. Then

$$
\left\langle F^{\prime}(v), v-u\right\rangle \geq \mu\|\xi(v, u)\|^{p}, \quad \forall u, v \in K .
$$

Proof. Let $F$ be a sharply higher order strongly pesudo convex function on $K$. Then

$$
F(v) \geq F(v+t(u-v))+\mu\left\{t^{p}(1-t)+t(1-t)^{p}\right\}\|\xi(v, u)\|^{p}, \quad \forall u, v \in K, t \in[0,1],
$$

from which, we have

$$
\frac{F(v+t(u-v)-F(v)}{t}+\mu\left\{t^{p-1}(1-t)+(1-t)^{p}\right\}\|\xi(v, u)\|^{p} \geq 0 .
$$

Taking limit in the above inequality, as $t \rightarrow 0$, we have

$$
\left\langle F^{\prime}(v), v-u\right\rangle \geq \mu\|\xi(v, u)\|^{p}, \quad \forall u, v \in K,
$$

the required result.

Definition 11. A function $F$ is said to be a pseudo convex function, if there exists a strictly positive bifunction $B(\cdot, \cdot)$, such that

$$
F(v)<F(u) \quad \Rightarrow \quad F(u+t(v-u))<F(u)+t(t-1) B(v, u),
$$

for all $u, v \in K, t \in[0,1]$.

Theorem 6. If the function $F$ is higher order strongly convex function such that $F(v)<F(u)$, then the function $F$ is higher order strongly pseudo convex.

Proof. Since $F(v)<F(u)$ and $F$ is higher order strongly convex function, then for all $u, v \in K, t \in[0,1]$, we have

$$
\begin{aligned}
F(u+t(v-u)) & \leq F(u)+t(F(v)-F(u))-\mu\left\{t^{p}(1-t)+t(1-t)^{p}\right\}\|\xi(v, u)\|^{p} \\
& <F(u)+t(a-t)(F(v)-F(u))-\mu\left\{t^{p}(1-t)+t(1-t)^{p}\right\}\|\xi(v, u)\|^{p} \\
& =F(u)+t(t-1)(F(u)-F(v))-\mu\left\{t^{p}(1-t)+t(1-t)^{p}\right\}\|\xi(v \cdot u)\|^{p} \\
& <F(u)+t(t-1) B(u, v)-\mu\left\{t^{p}(1-t)+t(1-t)^{p}\right\}\|\xi(v, u)\|^{p}, \forall u, v \in K,
\end{aligned}
$$

where $B(u, v)=F(u)-F(v)>0$, the required result.

We now discuss the optimality for the differentiable generalized strongly convex functions, which is the main motivation for our next result.

Theorem 7. Let $F$ be a differentiable higher order strongly convex function with modulus $\mu>0$. If $u \in K$ is the minimum of the function $F$, then

$$
F(v)-F(u) \geq \mu\|\xi(v, u)\|^{p}, \quad \forall u, v \in K .
$$

Proof. Let $u \in K$ be a minimum of the function $F$. Then

$$
F(u) \leq F(v), \quad \forall v \in K .
$$


Since $K$ is a convex set, so, for all $u, v \in K, t \in[0,1]$,

$$
v_{t}=(1-t) u+t v \in K \text {. }
$$

Taking $v=v_{t}$ in (15), we have

$$
0 \leq \lim _{t \rightarrow 0} \frac{F(u+t(v-u))-F(u)}{t}=\left\langle F^{\prime}(u), v-u\right\rangle .
$$

Since $F$ is differentiable higher order strongly convex function, so

$$
F(u+t(v-u)) \leq F(u)+t(F(v)-F(u))-\mu\left\{t^{p}(1-t)+t(1-t)^{p}\right\}\|\xi(v, u)\|^{p}, \quad \forall u, v \in K,
$$

from which, using (16), we have

$$
\begin{aligned}
F(v)-F(u) & \geq \lim _{t \rightarrow 0} \frac{F(u+t(v-u))-F(u)}{t}+\mu\left\{t^{p-1}(1-t)+(1-t)^{p}\right\}\|\xi(v, u)\|^{p} \\
& =\left\langle F^{\prime}(u), v-u\right\rangle+\mu\|\xi(v, u)\|^{p}
\end{aligned}
$$

the required result (14).

Remark 1. We would like to mention that if

$$
\left\langle F^{\prime}(u), v-u\right\rangle+\mu\|\xi(v, u)\|^{p} \geq 0, \quad \forall u, v \in K,
$$

then $u \in K$ is the minimum of the function $F$. The inequality (17) is called the higher order strongly variational inequality and appears to be a new one.

Theorem 8. Let $f$ be a higher order strongly affine function. Then $F$ is a higher order strongly convex function if and only if $H=F-f$ is a convex function.

Proof. Let $f$ be a higher order strongly affine function, then

$$
f((1-t) u+t v)=(1-t) f(u)+t f(v)-\mu\left\{t^{p}(1-t)+t(1-t)^{p}\right\}\|\xi(v, u)\|^{p},
$$

for all $u, v \in K$.

From the higher order strongly convexity of $F$, we have

$$
F((1-t) u+t v) \leq(1-t) F(u)+t F(v)-\mu\left\{t^{p}(1-t)+t(1-t)^{p}\right\}\|\xi(v, u)\|^{p},
$$

for all $u, v \in K$.

From (18) and (19), we have

$$
F((1-t) u+t v)-f((1-t) f(u)+t f(v) \leq(1-t)(F(u)-f(u))+t(F(v)-f(v)),
$$

from which it follows that

$$
\begin{aligned}
H((1-t) u+t v) & =F((1-t) u+t v)-f((1-t) u+t v) \\
& \leq(1-t) F(u)+t F(v)-(1-t) f(u)-t f(v) \\
& =(1-t)(F(u)-f(u))+t(F(v)-f(v)),
\end{aligned}
$$


which show that $H=F-f$ is a convex function. The inverse implication is obvious.

It is worth mentioning that the higher order strongly convex function is also Wright higher order strongly convex function. From the definition 2, we have

$$
\begin{aligned}
F(u+t(v-u))+F(v+t(u-v)) \leq & F(u)+F(v) \\
& -2 \mu\left\{t^{p}(1-t)+t(1-t)^{p}\right\}\|\xi(v, u)\|^{p},
\end{aligned}
$$

for all $u, v \in K, t \in[0,1]$, which is called a higher order Wright strongly exponentially generalized preinvex function. One can study the properties and applications of the Wright higher order strongly convex functions in optimization operations research.

\section{Application}

Bynum [19] and Chen et al. [20-22] have studied the properties and applications of the parallelogram laws for the Banach spaces. $\mathrm{Xi}$ [5] established new characteristics of $p$-uniform convexity and $q$-uniform smoothness of a Banach space using $\|\cdot\|^{p}$ and $\|\cdot\|^{q}$, respectively. These results can be obtained from the concepts of higher order strongly convex (concave) functions, which can be viewed as a novel application.

Setting $F(u)=\|u\|^{p}$ and $\xi(v, u)=v-u$ in Definition 2, we have

$$
\|u+t(v-u)\|^{p} \leq(1-t)\|u\|^{p}+t\|v\|^{p}-\mu\left\{t^{p}(1-t)+t(1-t)^{p}\right\}\|v-u\|^{p},
$$

for all $u, v \in K, t \in[0,1]$. Taking $t=\frac{1}{2}$ in (21), we have

$$
\left\|\frac{u+v}{2}\right\|^{p}+\mu \frac{1}{2^{p}}\|v-u\|^{p} \leq \frac{1}{2}\|u\|^{p}+\frac{1}{2}\|v\|^{p}, \forall u, v \in K,
$$

which implies that

$$
\|u+v\|^{p}+\mu\|v-u\|^{p} \leq 2^{p-1}\left\{\|u\|^{p}+\|v\|^{p}\right\}, \forall u, v \in K,
$$

which is known as the lower parallelogram for the $l^{p}$-spaces. In a similar way, one can obtain the upper parallelogram law as

$$
\|u+v\|^{p}+\mu\|v-u\|^{p} \geq 2^{p-1}\left\{\|u\|^{p}+\|v\|^{p}\right\}, \forall u, v \in K,
$$

Similar to Definition 3, we can have

$$
\|u+v\|^{p}+\mu\|v-u\|^{p}=2^{p-1}\left\{\|u\|^{p}+\|v\|^{p}\right\}, \forall u, v \in K,
$$

which is known as the parallelogram for the $l^{p}$-spaces. For the applications of the parallelogram laws in Banach spaces in prediction theory and applied sciences, see References [19-23] and the references therein.

\section{Conclusions}

In this paper, we have introduced and studied a new class of convex functions, which are called higher order strongly convex functions. We have improved the results of Lin and Fukushima [1]. It is shown that several new classes of strongly convex functions can be obtained as special cases of these higher order strongly convex functions. We have studied the basic properties of these functions. We have shown that one can derive the parallelogram laws in Banach spaces, which have applications in prediction theory and stochastic analysis. These parallelogram laws can be used to characterize the $p$-uniform convexity and 
$q$-uniform smoothness of a Banach spaces. The interested reader may explore the applications and other properties of the higher order strongly convex functions in various fields of pure and applied sciences. This is an interesting direction for future research.

Author Contributions: All the authors contributed equally, read and approved the final version.

Acknowledgments: The authors extend their appreciation to the International Scientific Partnership Program ISPP at King Saud University, Riyadh, Saudi Arabia for funding this research work through ISPP-0125. Authors are grateful to the referees for their valuable and constructive comments, which helped us to improve the presentation of the paper.

Conflicts of Interest: The authors declare no conflict of interest.

\section{References}

1. Lin, G.H.; Fukushima, M. Some exact penalty results for nonlinear programs and mathematical programs with equilibrium constraints. J. Optim. Theory Appl. 2003, 118, 67-80. [CrossRef]

2. Mishra, S.K.; Sharma, N. On strongly generalized convex functions of higher order. Math. Inequal. Appl. 2019, 22, 111-121. [CrossRef]

3. Polyak, B.T. Existence theorems and convergence of minimizing sequences in extremum problems with restrictions. Soviet Math. Dokl. 1966, 7, 2-75.

4. Karamardian, S. The nonlinear complementarity problems with applications, Part 2. J. Optim. Theory Appl. 1969, 4, 167-181. [CrossRef]

5. Zu, D.L.; Marcotte, P. Co-coercivity and its role in the convergence of iterative schemes for solving variational inequalities. SIAM J. Optim. 1996, 6, 714-726.

6. Nikodem, K.; Pales, Z.S. Characterizations of inner product spaces by strongly convex functions. Banach J. Math. Anal. 2011, 1, 83-87. [CrossRef]

7. Qu, G.; Li, N. On the exponentially stability of primal-dual gradeint dynamics. IEEE Control Syst. Lett. 2019, 3, 43-48. [CrossRef]

8. Awan, M.U.; Noor, M.A.; Du, T.-S.; Noor, K.I. New refinemnts of fractional Hermite-Hadamard inequality. RACSAM 2019, 113, 21-29. [CrossRef]

9. Adamek, M. On a problem connected with strongly convex functions. Math. Inequal. Appl. 2016, 19, 1287-1293. [CrossRef]

10. Angulo, H.; Gimenez, J.; Moeos, A.M.; Nikodem, K. On strongly $h$-convex functions. Ann. Funct. Anal. 2011, 2 , 85-91. [CrossRef]

11. Azcar, A.; Gimnez, J.; Nikodem, K.; Snchez, J.L. On strongly midconvex functions. Opuscula Math. 2011, 31, 15-26. [CrossRef]

12. Lara, T.; Merentes, N.; Nikodem, K. Strongly $h$-convexity and separation theorems. Int. J. Anal. 2016, 2016. [CrossRef]

13. Niculescu, C.P.; Persson, L.E. Convex Functions and Their Applications; Springer: New York, NY, USA, 2018.

14. Noor, M.A. Fundamentals of equilibrium problems. Math. Inequal. Appl. 2006, 9, 529-566. [CrossRef]

15. Noor, M.A.; Noor, K.I. On generalized strongly convex functions involving bifunction. Appl. Math. Inform. Sci. 2019, 13, 411-416.

16. Noor, M.A.; Noor, K.I. Higher order strongly generalized convex functions. Math. Inequal. Appl. 2019, 14. AVailable online: https:/ / www.researchgate.net/publication/335965531_Higher_Order_Strongly_Generalized_ Convex_Functions (accessed on 2 October 2019).

17. Oettli, W.; Thera, M. On maximal monontoncity of perturbed mapping. Bollettino dell'Unione Matematica Italiana 1995, 9, 47-55.

18. Pecric, J.; Proschan, F.; Tong, Y.I. Convex Functions, Partial Ordering and Statistical Applications; Academic Press: New York, NY, USA, 1992.

19. Bynum, W.L. Weak parallelogram laws for Banach spaces. Can. Math. Bull. 1976, 19, 269-275. [CrossRef] 
20. Cheng, R.; Harris, C.B. Duality of the weak parallelogram laws on Banach spaces. J. Math. Anal. Appl. 2013, 404, 64-70. [CrossRef]

21. Cheng, R.; Ross, W.T. Weak parallelogram laws on Banach spaces and applications to prediction. Period. Math. Hung. 2015, 71, 45-58. [CrossRef]

22. Cheng, R.; Mashreghi, J.; Ross, W.T. Optimal weak parallelogram constants for $L_{p}$ space. Math. Inequal. Appl. 2018, 21, 1047-1058.

23. $\mathrm{Xu}, \mathrm{H} .-\mathrm{K}$. Inequalities in Banach spaces with applications. Nonlinear Anal. TMA 1991, 16, 1127-1138. [CrossRef]

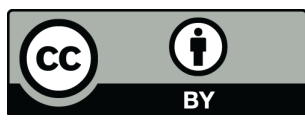

(C) 2019 by the authors. Licensee MDPI, Basel, Switzerland. This article is an open access article distributed under the terms and conditions of the Creative Commons Attribution (CC BY) license (http:/ / creativecommons.org/licenses/by/4.0/). 